\title{
MANAGING ARTIFICIAL INTELLIGENCE DEPLOYMENT IN THE PUBLIC SECTOR
}

Averill Campion (ESADE -Ramon Llull University), Mila Gasco-Hernandez (Rockefeller College of Public Affairs \& Policy and CTG UAlbany, University at Albany-SUNY), Slava

Jankin Mikhaylov (Data Science Lab, Hertie School), Marc Esteve (University College London and ESADE - Ramon Llull University) 


\section{ABSTRACT}

The scarcity of empirical evidence surrounding the organizational challenges and successful approaches to artificial intelligence (AI) deployment has resulted in mostly theoretical conceptualizations. By analyzing policy labs and offices of data analytics across the US to understand organizational challenges of AI adoption and implementation in the public sector as well as to identify successful management strategies to address such challenges, our study moves from speculation to gathering evidence. Our findings show that most challenges are found during the implementation stage and include challenges related to skills, culture, and resistance to share the data driven by data challenges. Further, our results indicate that long term strategies and short term actions need to be put in place to address these challenges. Among the first ones, leadership and executive support and stakeholder management seem to play an important role. Data standardization, training, and data-sharing agreements also seem to be successful specific shortterm actions.

\section{KEYWORDS}

Artificial intelligence adoption, artificial intelligence implementation, public sector, management, organizational challenges 


\section{MANAGING ARTIFICIAL INTELLIGENCE DEPLOYMENT IN THE PUBLIC SECTOR}

Most of the current debate on AI in the public sector revolves around potential benefits and areas of application ranging from providing solutions and improving performance in the form of predictions and alerts, calculations, analysis, and learning, to establishing knowledge management systems to retrieve and classify documents on a large scale and large scale audit activity (Gomes de Sousa et al. 2019), and augmenting police judicial decisions related to the resolution of crimes and crime prediction (Mikhaylov et al. 2018). In addition, several works have also looked at risks and areas of concern of AI projects, particularly taking into account privacy and security as well as ethical concerns (Wirtz et al. 2018). However, little has been said about the organizational challenges of adopting and implementing AI which may include, but not be limited to, resistance to share the data, lack of an AI strategy, and the need for staff specialization and expertise.

Yet, the study of organizational challenges in the development of AI in the public sector is key given the high costs incurred by governments in this endeavour. Only in the US, the federal government plans to spend almost $\$ 1$ billion in nondefense artificial intelligence research and development in fiscal year $2020^{1}$. The budget is four times more only for the US Department of Defense ${ }^{2}$. Other governments are devoting far more. The Chinese government, for example, is expected to invest more than $\$ 30$ billion in $\mathrm{AI}$ and related technologies and the city of Beijing

\footnotetext{
${ }^{1}$ See, for example, https://www.nextgov.com/emerging-tech/2019/09/administration-projects-agencies-will-spend1-billion-artificial-intelligence-next-year/159781/.

${ }^{2}$ See https://about.bgov.com/news/finding-artificial-intelligence-money-fiscal-2020-budget/.
} 
alone has already committed $\$ 2$ billion ${ }^{3}$. Thus, research is needed that examines organizational challenges and how governments are addressing them, so some essential leading practices can be gleaned that can result in successful AI projects with important academic and practical contributions.

In addition, although the literature recognizes the complexity of AI projects (Mikhaylov et al., 2018), it does not explore in-depth the non-technical causes of such complexity and the related challenges. On one hand, identification and prioritization of challenges of AI development in the public sector heavily depend on stakeholders' views. As a result, challenges can be both diverse and contradictory (Sun \& Medaglia 2019). For example, in the adoption of the AI system IBM Watson in public healthcare in China, IT firm managers involved identified important organizational challenges. Among others, they highlighted mainly a lack of an innovation spirit in the public health sector, but did not mention technological challenges; interestingly enough, for hospital managers/doctors, economic challenges, such as the expensive fees covered by patients to support Watson, were more important than other types of challenges when considering adoption of AI in healthcare (Sun \& Medaglia 2019). On the other hand, AI development in the public sector occurs in different stages that face different challenges (GDS AI Implementation Guide 2019). As in any innovation project (Rogers, 2003), there is an adoption stage where the decision to adopt $\mathrm{AI}$ is taken and there is an implementation stage that can also be divided into different phases. The first one, planning, involves considering the current data state, decisions about whether to build, buy or collaborate, allocate responsibility for AI, assessing existing data, building the AI team, getting the data ready for AI, and planning the AI modelling phase. The second one, alpha, involves building and evaluating the machine

\footnotetext{
${ }^{3}$ See https://www.marketwatch.com/story/china-is-overtaking-the-us-as-the-leader-in-artificial-intelligence-201902-27.
} 
learning model and the third one, beta, involves deploying and maintaining the model. Finally, there is also a diffusion stage, which involves the spread of AI throughout the organization or from one organization to another. Therefore, having a good understanding of challenges according to different perspectives (stakeholders involved) and stages of development may contribute to address the complexities of AI projects.

This paper aims to advance this debate by studying the organizational challenges of adoption and implementation of AI in the public sector as well as the existing successful management strategies to address those challenges. Therefore, our research questions are: 1) what are the organizational challenges of adopting and implementing AI in the public sector? and 2) what strategies are used to manage the organizational challenges of adopting and implementing AI in the public sector? For the purposes of this study, we follow OECD (2019), which defines an AI system as a "machine-based system that can, for a given set of humandefined objectives, make predictions, recommendations or decisions influencing real or virtual environments. It uses machine and/or human-based inputs to perceive real and/or virtual environments; abstract such perceptions into models (in an automated manner e.g. with ML or manually); and use model inference to formulate options for information or action. AI systems are designed to operate with varying levels of autonomy". Further, given that most public sector AI is still nascent (Sun \& Medaglia 2019), our study focuses on the adoption and implementation stages of AI development in the public sector.

\section{CHALLENGES OF AI IN THE PUBLIC SECTOR}

Current literature has classified challenges of AI in the public sector according to seven categories: 1) social challenges, 2) economic challenges, 3) technological challenges, 4) data 
challenges, 5) organizational and managerial challenges, 6) ethical challenges, and 7) political, legal, and policy challenges (Dwivedi et al. 2019; Sun \& Medaglia 2019).

Social challenges include issues related to existing societal norms and attitudes towards the adoption of AI in government. They may include unrealistic expectations towards AI technology, a societal misunderstanding of the capabilities of AI technologies, potential job losses, and increasing inequality. Economic challenges include obstacles concerning profitability and economic sustainability and, therefore, have to do with the costs and investments associated with the adoption of AI by public organizations. In addition, challenges may also be technological, that is, related to the nature and characteristics of AI technologies. Challenges under this category include the lack of transparency of AI algorithms, the difficulties of AI systems in processing unstructured data, and the lack of interoperability.

Since AI applications are heavily data-dependent, challenges associated with the data, such as the insufficient size of the available database, the absence of data standards, the difficulties of data integration, and mainly, data privacy and security concerns, have been often addressed in the literature. Ethical challenges, based on the impact of AI on moral principles, and political, legal, and policy challenges, such as obstacles connected to responsibility when errors occur using AI systems and the lack of policy regulations, have also been of much concern for both academics and practitioners.

Finally, organizational challenges are those related to each organization's strategy, human resources, and management practices in the adoption of AI. A few previous studies have timidly mentioned them, although they have not elaborated on them nor analyzed their impact on AI success. Sun and Medaglia (2019) identify two challenges of this nature: organizational resistance to share the data and the lack of in-house AI talent. The first one is particularly 
important because it raises additional issues such as the dilemma about who owns the data or the tension between the need for data integration and the interest of individual organizations. It also shows the importance of trust among stakeholders in the adoption of AI. Additional organizational challenges may include the lack of clear leadership, the lack of a strategy regarding adoption of $\mathrm{AI}$, the lack of $\mathrm{AI}$ deployment organizational guidelines, including criteria to standardize the way data is collected and shared, and the ease of use of AI applications (Dwivedi et al. 2019; Sun \& Medaglia 2019. Further, Krishnamurthy and Desouza (2018) refer to the importance of cultivating a culture of cross-agency collaboration, adopting crowd-centric approaches, and developing collaborative leadership and management support.

\section{ADOPTION AND IMPLEMENTATION OF AI IN GOVERNMENTS ACROSS THE US}

The main objective of this article is to explore the organizational challenges of adoption and implementation of $\mathrm{AI}$ in the public sector as well as the existing successful management strategies to address those challenges. This is novel because it moves from conceptual studies of the different types of challenges of AI projects in the public sector to empirical ones, and towards identifying actionable management strategies to address those challenges. To answer our research questions, we build on data collected through 19 in-depth interviews from policy labs and offices of data analytics (ODAs) across the US involved in AI adoption and implementation in the public sector at the local level of government.

Given our lack of specific knowledge on the number of organizations adopting and implementing AI in the US, we used purposeful sampling, a useful technique to identify and select information-rich cases for the most effective use of limited resources (Patton 2002). We did so by conducting an environmental scan, mainly using Google.com, which resulted in 11 
local public organizations. Then, and given that purposeful sampling involves identifying and selecting individuals or groups of individuals that are especially knowledgeable about or experienced with the phenomenon of interest, we contacted several individuals in the identified organizations and received 19 replies from 10 out of the 11 originally identified organizations, showing availability and interest to participate in our study. These 19 individuals belonged to five policy labs and five offices of data analytics at the local level, and contained several key areas of similarities to assist in strengthening our ability to analyze the organizational challenges. First, the policy labs and ODAs identified have been leaders in evidence based policy making and the creation of advanced data science informed services in local government. Second, both the policy labs and ODAs require intra/inter collaborative approaches to projects and have similar goals of promoting the use of data to enhance decision making abilities in government. Third, projects use political leadership when available, but many projects are ad-hoc in nature. Fourth, they are similar in terms of membership size (around 6 people per team in ODAs and 10 per team in policy labs) and organizational characteristics (ODAs tend to be located in the mayor's office and policy labs are run by universities with a reputation for data science excellence). Policy labs are collaborative entities formed to leverage academic expertise and university oriented skills to assist government organizations with the capacity and capability to adopt AI and data science informed policies and services; offices of data analytics (ODAs) are offices within city-level and/or regional government that drive internal adoption of AI and data science.

The interviews were conducted between January and April 2019 and included chief data scientists, chief data officers, project managers, partnership managers, assistant directors, data analysts, academics, and knowledge directors from both types of organizations. The main themes 
covered in the interviews included but were not limited to purpose (e.g., what is the mission of this organization?, what was the goal of adopting AI?), challenges (e.g., what challenges have you encountered in the process of adopting and implementing AI?, what are some of the risks that you still envision?), results (e.g. what have you achieved so far?, what has AI helped addressed?), and management strategies to overcoming challenges of AI projects in the public sector (e.g., given the challenges that you have described, what did you do to address them?, in your opinion, what are some important successful that should always be taken into account when adopting and implementing AI?). Interviews lasted between 30-45 minutes and were recorded and transcribed. The results were reported taking into account the confidentiality and anonymity guaranteed to the interviewees.

In general, we find that although different organizations have already taken the decision of adopting AI, they find themselves at different stages of implementation, therefore facing different challenges. Our results also indicate that the planning subphase is probably the one where more decisions need to be made to guarantee the success of AI in the public sector. In this respect, our interviews reveal that, during this subphase, challenges are importantly related to the resistance to share the data and require management approaches that encompass the wide ranging needs of a data oriented culture change, political support, and stakeholder management. Further, our results show that adoption and, mainly, implementation challenges are affected by the choice and combination of technique and topic, and that those choices have different implications for the strategies needed to drive success in the development of AI applications. The following paragraphs give further detail about these findings.

\section{The challenges of AI implementation}


Although the organizations that we addressed were already implementing projects, there was agreement on the fact that the decision to adopt AI was mainly a political or a policy-oriented decision that was made at the highest level of the organization. As a result, the main challenge identified in this first stage had precisely to do with leadership and having the political support: “if it's a mayoral initiative or something the city councilors are really wanting to see then there will be a lot more internal pressure and support for that to happen." Yet, given the collaborative nature of AI projects, having the right partners was also important. According to one of our interviewees: "in [name of city], we are very lucky because there is a lot of buy in for that in the city and we've had some super strong relationships and partners across the city. So it's worked out pretty well for us."

Although our informants had already taken the decision of adopting AI, they were still at the implementation stage. Actually, most of them were still getting ready for AI deployment, given the many challenges they were experiencing, particularly in terms of skills (data illiteracy and a general lack of awareness about how to think about applying AI techniques to solve problems), collaborative culture (challenges associated with a lack of cooperation among units and/or external organizations), and resistance to share the data driven by data challenges. Skills. Our interviewees referred to the need of being aware about how to think about applying AI techniques to solve problems and therefore about the ability to ask data-oriented questions. An interviewee from an ODA pointed out, "we have other folks who have no clue where to begin. Either because they have no data literacy, or because no one has documented what this is supposed to mean; or because someone else decided this is the indicator of success, and they don't necessarily believe that." Another respondent stated, "sometimes they don't know the right questions to ask of their organization about what data is because sometimes they 
confuse performance information they have with data.” In addition, a Chief Data Officer told us that, "I had a conversation with one department where they didn't understand that, while they had made their data available in graph form, the underlying data, the raw data, is very valuable." Moreover, to develop AI projects in some cases requires that people in organizations have a basic understanding of what a data-oriented question is that can be solved using an AI technique. As another interviewee stated, "there is also the matter of trying to train the higher levels in the organizations so they can ask the right sets of questions and be involved in pushing forward these types of projects." For example, if there are priorities around certain topics like green spaces, or mental health then,

"If the higher levels are thinking, not just in terms of policy documents, but analytical questions which could be answered by AI techniques, even if they wouldn't know how to do it themselves... if they could understand the kinds of things that need to be asked, then suddenly there is a lot more power and motivation."

Although this also seems to point at an issue relevant for the adoption phase, our interviewees found it was much more relevant during the implementation phase, once the work around using AI to address specific public problems starts.

Collaborative culture. As discussed, often, AI projects are collaborative in nature, which means that obtaining the extent of data needed for deploying an AI project may require data sharing among units within the same organization or among different organizations; yet, organizations have to be willing to cooperate in order to share the data. Our findings show that the lack of a collaborative culture, based on data-sharing, is a key challenge during the first phase of the implementation process. In addition, our findings indicate that the history of past collaborations and the lack of trust play a major role in shaping such culture. In the words of one 
of our interviewees: “a lot of government employees have been here a long time and they've been burned in the past with data sharing and don't want to get burned again." Another one stated: "the biggest risk aversion I encounter is in the sharing of the data. The agencies that are more risk averse is because of a lack of certainty about how much data to share and whether they feel comfortable sharing the data." Yet, another one conveyed: "what I don't want to do is get tied up in information sharing agreements and end up with issues you can't resolve. For instance, sensitive information that the police might hold, they might not want to share with a local authority and vice versa." Further: "I think a lot of times a lack of trust or level of risk aversion comes from an area of uncertainty."

\section{Resistance to share the data driven by data challenges. Most of our interviews}

recognized that, although they knew that they had to share data with other organizations for AI to be successful, that was not always easy given some important features of the data and, therefore, data challenges. For example, although most of our interviewees referred to the importance of data integration, they also agreed that AI projects require a lot of data which may result in availability, but, also, quality issues. In terms of availability, AI projects do not only use internal data. One of our interviewees said: "as a city, we are part of an agreement where we purchase high quality, sensor data; satellite imagery with the county — so there are many places where you can get access to really complex information that just a few years ago we weren't able to tap into". This adds complexity to the process of collecting data from which patterns will emerge to be leveraged to make predictions for future decisions. In addition, data quality, which includes aspects such as consistency, integrity, accuracy, and completeness, is also key, particularly when the data needs to be shared and integrated. In addition, our interviewees also referred to the criticality of privacy issues: "they also want to be sure that since they are the stewards of this 
public data, that privacy isn't getting compromised. So, two sides of the same coin: one is a legitimate fear about privacy and one is a fear about organizational reputational risks".

\section{Making AI implementation work}

Despite the challenges identified, the different organizations of our sample put several strategies in place to address them. These strategies included data standardization, training, and datasharing agreements. In addition to these specific actions, political will and executive support as well as stakeholder management proved essential in managing the overall implementation process.

Data standardization. Our interviewees agreed on the need to address resistance to share the data by taking action about data quality issues, which included a standardized set of guidelines and procedures for data quality. This could result in a formal process of moving away from fragmented quality standards held by various people and organizations towards more systematic rules and frameworks. For example, one of the ODAs created a data quality guidebook to enhance data quality challenges for public organizations across the city. The guidebook provides steps for obtaining better quality such as collection needs and requirements, how to define the data set, and how to define the set of policies and processes to manage the data through its lifecycle.

Training. Although training and skill development has been identified by the literature as an important strategy to successfully implement AI, the training our interviewees referred to had more to do with awareness capabilities and a strategic understanding of the data. In this respect, some of them stated that what mattered was to train high level staff in government in how to turn a challenge into the appropriate data-based question. One interviewee explained: "a city 
challenge for us at the moment is improving air quality... How does that translate into a series of questions for our data? Both in terms of the data that we have... and the data that we do not have. And that process of making the questions appropriate is really important." Another one shared that he was working across the city to "target policy folks within the city that are not IT people, and help them understand how to better leverage their data; how to better format questions so that you're answering them with data."

Data sharing agreements. There was wide consensus on the usefulness on data-sharing agreements to build trust among units and/or organizations involved in an AI project and, therefore, to increase the willingness of sharing the data. One of our interviewees explained: "it's been mostly as a tool to formalize in writing that this is what we're going to do; this is what we're not going to do. Just to make that stakeholder feel better." Interestingly enough, our interviewees also emphasized the importance of informal relationships, particularly before the agreement signature: "in the process of setting up a data sharing agreement, I think that showing that you have ideas, and not waiting until that agreement is signed, to get things kick-started, can be helpful too." Another one said: "I think more often than not, you have to build some trust before you start with the institutional agreements. Then once you have that formal agreement, in place, that's a symbol." In addition: "a lot of the first step is getting those people to really trust each other, and understand each other. And then that is a good foundation for being able to figure out a foundational agreement and governance structure." Further, there were references to the role of inter-personal relationships: "it's important to start with the inter-personal side of things and get people really comfortable and I think that it is really hard to tell anyone that they have to do anything. You have to start at a very human level." 
Political will and executive support. When possible, political and executive sponsorship, both informal and formal, provided the necessary legitimacy to push an initial AI project forward, according to informants. AI tools can change the process of how agencies make service decisions, so higher level mandates prove useful. For example, commenting on the process change of using a predictive analytics tool to help better prioritize decisions around services for the homeless, one manager stated "And there was a change where we were prioritizing [now] the most needy people, who are harder to serve. There was an adjustment overall for people to say: 'This will be harder" but people recognized they were having to do it because it was best to serve the right people and it came from a higher level." Reflecting on the development of automated dashboards for a city government, one manager commented: "we were able to stand them up pretty quickly. This was a special case because you had a new mayor saying, 'I want it like this as soon as possible,' so the departments were pretty eager and willing [to move forward]." Interestingly, pursuing political mandates and executive pushes may not always be a viable long term strategy. Many respondents commented, "We have to play a balanced role because, being placed in the Mayor's office, we have power to convene and also power to dictate what departments focus on. But we've been avoiding implementing that power on the latter, mostly because we don't think we are there yet in terms of the trust with what data can achieve and we don't want to exclusively tell departments what exactly they need to do."

Stakeholder management. As previously stated, our findings show that, often, AI development involves collaborating with other organizations because AI projects may cut across many fields, such as domestic abuse and child welfare. Thus, identifying the stakeholders of an AI project and planning and implementing actions designed to formally and informally engage with them becomes an important management strategy: "making formal relationships you 
coalesce around helps ODAs survive and thrive... It is important to have a formal function and vision..., but it is about creating informal connections that ensure the culture change happens and that we get some interest in and exciting work that can engage and galvanize people." In this respect, our interviewees identified several types of stakeholders: the AI team, which usually includes one or more data scientists, the organizations that have the data that is needed (or data providers), the beneficiaries (usually the citizens), and additional partners. Interestingly enough, there was agreement on the usefulness of face-to-face meetings to engage with stakeholders. One interviewee stated: "face-to-face always help. We try to meet as regularly as possible. Our teams try to go there, especially when we're building a tool, we'll review it with them, take their feedback, build it in. So that face-to-face interaction really helps enable an open dialogue and feedback." Another informant explained: "we've done focus groups with families, with community leaders, and the response we get is 'we don't care how you identify us, as long as you help us, and do the right thing." 'Additional strategies included having interviews at the beginning of the project ("we have a couple of sites where they were working to build a new integrative data system and they actually went systematically from agency lead to agency lead and did qualitative interviews to understand the context in which these agencies work, to understand what data sharing is already occurring, and to understand what value could be added for that agency head, so that moving forward on the initial demonstration project, they could choose some that really added value for many of the stakeholders involved) and signing MoUs ("we've seen that done in a variety of ways: it could be done in an enterprise level of an MOU; or in a charter document; but I think being explicit upfront about uses is important").

\section{A HOLISTIC MANAGEMENT APPROACH TO AI DEVELOPMENT}


Our findings provide answers to our two research questions. Regarding the first one, we found that during the adoption stage, the level of political support clearly conditions the decision to adopt AI. In addition, three important challenges, skills, a lack of a collaborative culture, and resistance to share the data driven by data challenges hinder the implementation process, particularly during the planning subphase. Regarding the second research question, we identified two long-term strategies, political and executive sponsorship and stakeholder management, and three specific short term actions, data standardization, training, and data-sharing agreements. Political will and executive support proved important along the whole process of AI development, and particularly during the implementation stage. Stakeholder management was also considered a good strategy to put in place that began with the identification of stakeholders, probably during the adoption stage, and aimed at engaging them through formal and informal actions over the implementation stage.

Our findings and answers to our research questions also provide additional conclusions. First, they show that, currently, most challenges of AI adoption in the public sector are manifested during the implementation stage and, specifically, during the first subphase of planning. This indicates that AI initiatives in the public sector are still at an early stage of development and confirms that innovation in the public sector takes place at a slow pace. Further, it shows that public organizations are eager to identify and encourage needed innovations (such as AI), but that, given the planning implementation challenges they encounter, they struggle to move towards the alpha and beta implementation subphases and, moreover, to the diffusion/institutionalization stage. Further research would therefore be needed to widen our understanding of challenges that take place during the alpha and beta subphases of the implementation stage as well as how different contexts, such as the maturity of the technology, 
the reasons why organizations adopt new technologies, and the differences between hard and soft policy domains, influence those challenges. Further, new studies could also look at challenges during the diffusion stage, which is important because as, previous literature has already indicated (e.g. DeVries et al., 2016; Baltazar, 2015), the benefits of innovation are most powerful when organizational systems and structures can institutionalize such innovation.

Second, despite the importance given to non-technical challenges over technical and datarelated challenges by the literature on digital government and information technology (IT) in public organizations, our findings show that data challenges seem to be particularly relevant in AI projects given the risks associated to these projects, especially in terms of data availability and quality, which, in addition, condition data sharing and integration. We therefore conclude that the nature and characteristics of AI projects in the public sector, which are technological, collaborative, and based on heavy data sharing, determine the prevalence of certain risks and concerns, which would not be found in other types of innovations developed in the public sector. We argue that data and organizational challenges actually reinforce each other, which results in difficulties during the implementation process, compromising the success of AI projects. Further studies should look at the specific interactions among these challenges and, therefore, at the direct and indirect effects on the success of AI projects.

Third, our results indicate that, although most management strategies and actions were focused on overcoming issues related to the preparation of AI deployment, efforts were still being made to create the stability needed for a more long term vision of AI development. In fact, this combination of both a project-oriented focus, and a broader, strategic-oriented focus were simultaneously entwined with one another: often, data challenges were being solved with a combination of informal, relationship-based management activities, as well as with more formal 
data-related solutions. In order to address the immediate challenges of data-related concerns about availability, quality, integration and the lack of skills in public organizations, management activities leveraged formal mechanisms like data standardization, training, and data sharing agreements. However, building long term cooperation also required increasing trust at the individual level in public organizations. We therefore argue that balancing long term with short term strategies is key in addressing implementation challenges and that leadership plays a key role in doing so. Short term actions contribute to address specific problems that paralyze dailybasis operations while long term strategies provide legitimacy, stability, and sustainability of AI development. In addition, short terms actions make quick wins possible, which are critical for maintaining momentum to keep AI projects, which are costly and complex, moving forward. Further, we conclude that such strategies should include both formal and informal actions. More systematic research is therefore needed to better understand how organizations are combining short and long terms approaches as well as formal and informal mechanisms to overcome the challenges of AI development.

Figure 1 shows the conceptual framework derived from the findings that illustrate the combination of success factors for theoretical development around the management of AI development challenges in the public sector. 
Figure 1: Managing AI deployment in the public sector

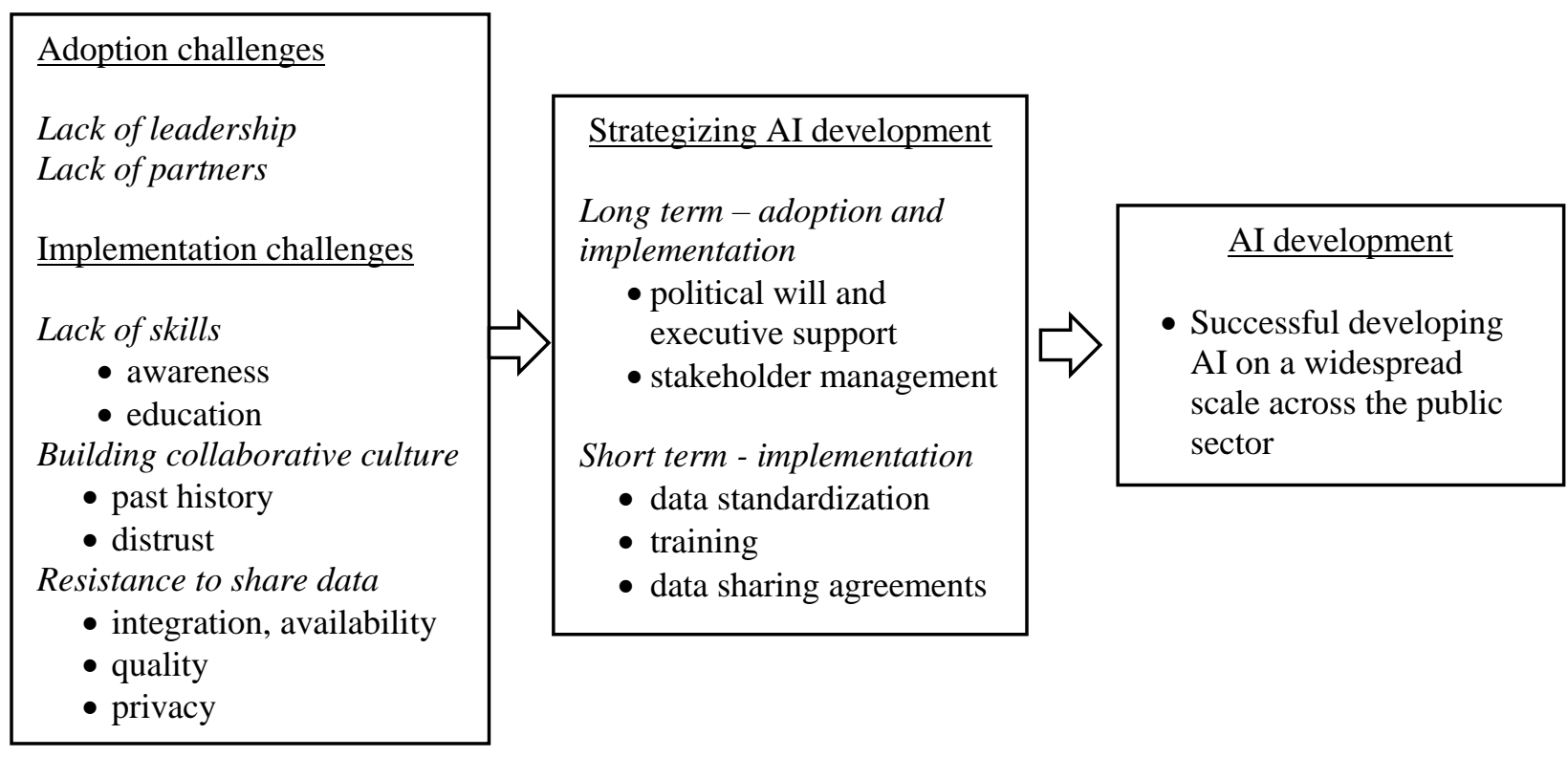

\section{CONCLUSION}

The lack of research on AI development in the public sector has resulted in a limited understanding of the organizational challenges and lessons learned from real deployment efforts. While some research has done well to describe the transformation AI will give rise to in the public sector in terms of, among other, workforce, policy making, and public services, the scarce knowledge about the organizational challenges faced at different stages of AI development as well as about successful approaches for addressing them is notorious. Therefore, more empirical research on such challenges and about strategies for managing the nuances of these challenges across different stages is needed. Our study contributes to this research and practical gap. Based on our findings, we present a framework for gauging challenges and stages of development in order to open up new paths when considering the management of public sector AI. A limitation to our findings is related to generalizability, as AI adoption is context specific and rooted in the dynamics of political embeddedness of the institutions enabling the project to occur. 
Nonetheless, we believe our research generates interesting results and further stimulates the conversation about AI deployment in the public sector.

\section{REFERENCES}

Baltazar, M. E. (2015). Creating competitive advantage by institutionalizing corporate social innovation. Journal of Business Research, 87(7), 1468-1474.

DeVries, H., Tummers, L. \& Bekkers, V. (2015). Innovation in the public sector: A systematic review and future research agenda. Public Administration, 94(1), 146-166.

Dwivedi, Y. K., Hughes, L., Ismagilova, E. et al (2019). Artificial Intelligence (AI): Multidisciplinary perspectives on emerging challenges, opportunities, and agenda for research, practice and policy. International Journal of Information Management, available online.

Gomes de Sousa, W., Pereira de Melo, E. R., De Souza Bermejo, P. H., Souza Farias, R. A. \& Oliveira Gomes, A. (2019). How and where is artificial intelligence in the public sector going? A literature review and research agenda. Government Information Quarterly, published online 25 July 2019.

Krishnamurthy, R. \& Desouza; K. (2014). Big data analytics: The case of the social security administration. Information Polity, 19(3), 165-178.

Mikhaylov, S. J., Esteve, M., \& Campion, A. (2018). Artificial intelligence for the public sector: opportunities and challenges of cross-sector collaboration. Philosophical Transactions of the Royal Society A: Mathematical, Physical and Engineering Sciences, 376(2128), 20170357.

OECD (2019). Artificial Intelligence in Society. Paris: OECD Publishing. 
Patton, M. (2002). Two decades of developments in qualitative inquiry: A personal, experiential perspective. Qualitative Social Work, 1(3), 261-283.

Rogers, E. M. (2003). Diffusion of Innovations. $5^{\text {th }}$ edition. New York, NY: Free Press.

Stevenson, A. (2013). The Public Sector: Managing the Unimaginable. Kogan Page Ltd.

Sun, T. Q. \& Medaglia, R. (2019). Mapping the challenges of artificial intelligence in the public sector: evidence from public healthcare. Government Information Quarterly, 36(2), 368383.

Wirtz, B., Weyerer, J., Geyer, C. (2019). Artificial intelligence and the public sectorapplications and challenges. International Journal of Public Administration, 42(7), 596-615. 